\title{
Assessment of color Doppler ultrasonography findings in gynecomastia
}

\author{
Ruken Yuksekkaya ${ }^{1}$, Fatih Celikyay $^{1}$, Mustafa Ozcetin $^{2}$, Mehmet Yuksekkaya $^{3}$, Yasemin Asan ${ }^{4}$
}

${ }^{1}$ Gaziosmanpasa University Radiology Department, ${ }^{2}$ Bulent Ecevit University, Pediatrics Department, Zonguldak, ${ }^{3}$ Department of Biomedical Engineering, Baskent University, Ankara, ${ }^{4}$ Gaziosmanpasa University, Pediatrics Department, Tokat, Turkey

\begin{abstract}
Aim: To evaluate the color Doppler ultrasound (US) findings of gynecomastia and compare them with sonographic Tanner staging to determine an additional diagnostic tool. Patients and methods: Eighteen male (mean age 13.87 \pm 1.72 ) patients (36 breasts) with breast enlargement were examined with gray-scale and color Doppler ultrasound (US), and these were used to determine the Tanner stages. In addition, the arterial and venous flow scores were assessed. The resistivity index (RI) values were also measured at the medial, lateral, and retroareolar regions of the breasts, and the mean RI values for each breast were then calculated. Results: Gynecomastia was detected in 30 breasts, and the mean retroareolar thickness was $8.03 \mathrm{~mm}$. Furthermore, arterial and venous flows were detected in $73.3 \%$ and $56.7 \%$ of the breasts, respectively. However, there was no correlation between the arterial and venous flows and the retroareolar glandular tissue thickness. The average of the mean RI values of the three regions of the breast was 0.62 , and there was a statistically significantly positive correlation between the mean RI values of the breasts and the Tanner stages as established by US. Furthermore, there was a strongly significant positive correlation between the arterial and venous flow scores and the Tanner stages. Conclusions: Our research indicates that vascularity is correlated with breast development in patients with gynecomastia. Additionally, we determined that RI values might be as important as Tanner stages on US.
\end{abstract}

Keywords: Color Doppler ultrasound, gynecomastia, resistivity index, Tanner staging

\section{Introduction}

Gynecomastia is a common condition of the male breast [1], and it is characterized by palpable, mobile, subareolar tissue that can be found on clinical examination. There is little information regarding the use of color Doppler ultrasonography (US) to evaluate this condition. The effect of vascularity in the development of gynecomastia is unknown. Our aim was to evaluate the color

Received 26.06.2013 Accepted 08.09.2013

Med Ultrason

2013, Vol. 15, No 4, 285-288

Corresponding author: Ruken Yuksekkaya, Assistant Professor Gaziosmanpasa University Radiology Department, 60100 Tokat, Turkey.

Phone: + 905327174634

Fax: + 903562129417

E-mail: rukenyuksekkaya@yahoo.com
Doppler US findings with regard to gynecomastia to establish whether this could be a viable diagnostic tool.

\section{Patients and methods}

Adolescent and teenage male patients who underwent breast US at our department between March 2010 and January 2011 for the evaluation of gynecomastia were included for this prospective study. Neonatals were excluded from the study because it is difficult to evaluate neonatal breast tissue with color Doppler US, and adults were also not included because the atherosclerotic risk factors may influence the color Doppler US parameters. In addition to those two patient groups, those with pathological gynecomastia associated with a chromosomal disorder (i.e. Klinefelter syndrome), an underlying disease (i.e. a testicular tumor orhyperthyroidism), or a breast mass detected by gray-scale US were also excluded. The 
local Ethics Committee approved the study, and all participants and their parents gave their written informed consent prior to the investigation.

The study group consisted of 18 male (range 11-18 years; mean age $13.87 \pm 1.72$ ) patients with breast enlargement on physical examination. Thirty-six breasts were examined via gray-scale and color Doppler US by a radiologist with nine years of experience in this area. An ACUSON Antares US system (Siemens AG, Erlangen, Germany) with a 13-5 MHz linear array transducer was used. The examinations were performed with patients in the dorsal decubitus position. Gray-scale US was utilized to scan the breasts on both transverse and longitudinal planes to detect gynecomastia, and retroareolar glandular tissue thickness was also measured. Tanner staging was then evaluated according to the sonographic characteristics described by Garcia et al [2]. Next, the color Doppler examination was performed. The transmitted Doppler frequency ranged from 5 to $10 \mathrm{MHz}$, the pulse repetition frequency varied from 488 to $1563 \mathrm{~Hz}$, and the wall filter was always set at its lowest setting. Doppler gain was also adjusted to maximize sensitivity without producing color noise, and the angle correct cursor was manually aligned parallel to the flow direction, with Doppler angles of approximately 60 degrees. An axial gate size of $0.5 \mathrm{~mm}$ was used. The presence of arterial and venous flow via color Doppler US was evaluated at three regions of the breast, and spectral Doppler US was then performed to differentiate arterial and venous flows. Arterial flow was based on the number of arteries in which the flow was observed, and the scores were as follows: $0=$ no arterial flow, $1=$ arterial flow seen in one vessel, $2=$ arterial flow seen in two vessels, $3=$ arterial flow seen in three or more vessels. Venous flow was also scored using the same system. The resistivity index (RI) was measured at the horizontal line that crosses the nipple and at the medial, lateral and retroareolar regions of the breasts. The retroareolar region was the breast tissue at the posterior of the areola, and the other regions were the contiguity regions which were sited at the medial or lateral of the areola. The mean of the measured RIs for each breast were calculated.

Continuous data was expressed as mean \pm standard deviation (SD), and categorical data was given as numbers with related percentages (n, \%). The differences in the categorical data were analyzed using a chi-square test and a two-tailed $p$-value of $<0.05$ was considered to be statistically significant. The correlations between the arterial and venous flow and the retroareolar glandular tissue thickness along with the mean RI values were analyzed using Pearson's rank correlation coefficient. A correlation was considered to be present when the $p$ value for the difference was less than 0.05. All statistical analyses were performed with the SPSS version 11.01 for Windows software program (SPSS Inc, Chicago, IL, USA).

\section{Results}

There were 30 cases of gynecomastia in the 36 breasts, and it was bilateral in 12 of the patients. The retroareolar thickness was $8.03 \pm 5.33 \mathrm{~mm}$ (range $3-25 \mathrm{~mm}$ ). Arterial flow was detected in $22(73.3 \%)$ of the cases, and 17 $(56.7 \%$.) had venous flow. Seven patients had an arterial flow score of 1 (23.3\%), two had a score of $2(6.7 \%)$, and 13 had a score of $3(43.3 \%)$, and this was most commonly observed in the retroareolar $(\mathrm{n}=15,50 \%)$ region. In addition, arterial flow was found in the medial region in $13(43.3 \%)$ breasts and in the lateral region in 13 others (43.3\%). Regarding the venous flow scores, six patients had a score of $1(20 \%), 10$ had a score of $2(33.3 \%)$, and one patient had a score of $3(3.3 \%)$. The average mean RI values of the three breast regions containing vascularity was $0.62 \pm 0.084$ (range $0.47-0.83$ ), with values of $0.66 \pm 0.11$ in the medial region, $0.58 \pm 0.10$ in the lateral region, and $0.64 \pm 0.12$ in the retroareolar region. There was no correlation between the arterial and venous flows and retroareolar glandular tissue thickness ( $\mathrm{p}$ $>0.05)$. Five $(16.7 \%)$ breasts were in Tanner stage II, eight $(26.7 \%)$ were in Tanner stage III, $10(33.3 \%)$ were in Tanner stage IV, and seven (23.3\%) were in Tanner stage $\mathrm{V}$, and there was a positive correlation between the mean RI values of the three breast regions and the Tanner stages $(r=0.41, p=0.439)$. Moreover, the arterial and venous flow scores were strongly positively correlated with the Tanner stages $(\mathrm{r}=0.756 ; \mathrm{p}=0.000$ and $\mathrm{r}$ $=0.506 ; \mathrm{p}=0.04$, respectively) (table I and II, fig 1-5).

\section{Discussion}

Gynecomastia represents the proliferation of the ductal and glandular components of the male breast [3]. In neonates, it is accepted as a physiological event due to the influence from maternal estrogens across the placenta. During puberty, the peak frequency usually occurs between the ages of 13 and 14, with as many as $60 \%$ of male adolescents being affected [4]. Gynecomastia can also be associated with obesity, pathological conditions (i.e. Klinefelter syndrome, anarchism, acquired testicular failure, receptor defects, and hyperthyroidism), and drugs (anabolic steroids, digitalis, isoniazid, tricyclic antidepressants, and marijuana). It can also occur as a result of an imbalance between estrogen and androgen [2,5]. Gynecomastia is staged according to the Tanner grading system using clinical findings and US. 


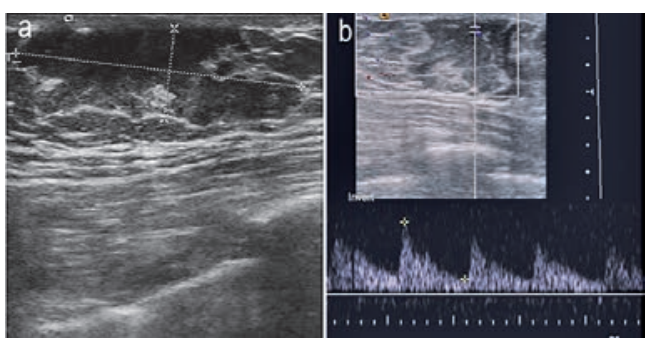

Fig 1. Gray scale (a) and duplex ultrasonography (b) of the right breast of a 14-year-old male patient, Tanner stage $\mathrm{V}$, with gynecomastia with an arterial flow score of 3 and a venous flow score of 2 .

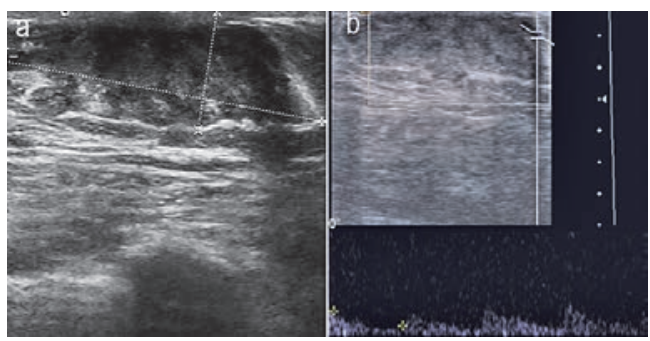

Fig 2. Gray scale (a) and duplex ultrasonography (b) of the left breast of the same patient as in fig 1 (Tanner stage $\mathrm{V}$ ) with an arterial flow score of 3 and a venous flow score of 2 .
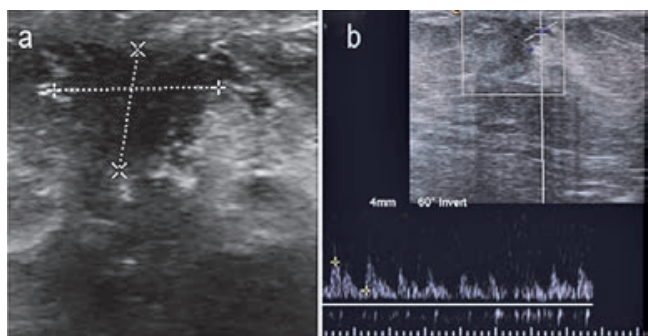

Fig 3. Gray scale (a) and duplex ultrasonography (b) of a 13-year-old male patient, Tanner stage III, with gynecomastia with an arterial flow score 2 and a venous flow score of 1 .

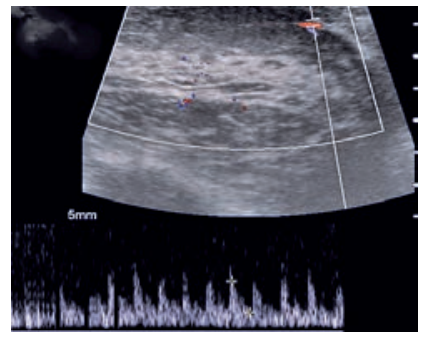

Fig 4. Duplex ultrasonography of a 15-year-old male patient, Tanner stage $\mathrm{V}$, with gynecomastia with an arterial flow score of 3 and a venous flow score of 2 .

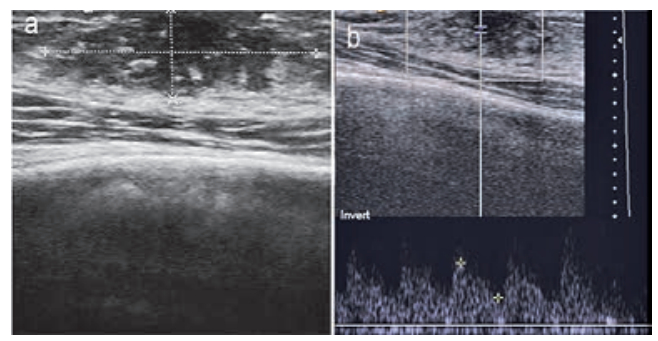

Fig 5. Gray scale (a) and duplex ultrasonography (b) of the right breast of a 13-year-old male patient, Tanner stage IV, with gynecomastia with an arterial flow score of 3 and a venous flow score 2 .

Table I. Distribution of arterial flow scores according to the Tanner stages

\begin{tabular}{|c|c|c|c|c|c|}
\hline $\begin{array}{l}\text { Arteri- } \\
\text { al flow } \\
\text { score }\end{array}$ & $\begin{array}{c}\text { Tanner } \\
\text { II } \\
(n=5)\end{array}$ & $\begin{array}{c}\text { Tanner } \\
\text { III } \\
(n=8)\end{array}$ & $\begin{array}{c}\text { Tanner } \\
\text { IV } \\
(\mathrm{n}=\mathbf{1 0})\end{array}$ & $\begin{array}{c}\text { Tanner } \\
\text { V } \\
(n=7)\end{array}$ & $\begin{array}{c}\text { Total } \\
(n=30)\end{array}$ \\
\hline 0 & 3 & 5 & & & $8(26.7 \%)$ \\
\hline 1 & 2 & 1 & 4 & & $7(23.3 \%)$ \\
\hline 2 & & 1 & 1 & & $2(6.7 \%)$ \\
\hline 3 & & 1 & 5 & 7 & $13(43.3 \%)$ \\
\hline
\end{tabular}

Table II. Distribution of venous flow scores according to the Tanner stages

\begin{tabular}{|c|c|c|c|c|c|}
\hline $\begin{array}{c}\text { Venous } \\
\text { flow } \\
\text { score }\end{array}$ & $\begin{array}{c}\text { Tanner } \\
\text { II } \\
(n=5)\end{array}$ & $\begin{array}{c}\text { Tanner } \\
\text { III } \\
(n=8)\end{array}$ & $\begin{array}{c}\text { Tanner } \\
\text { IV } \\
(\mathbf{n}=\mathbf{1 0})\end{array}$ & $\begin{array}{c}\text { Tanner } \\
\text { V } \\
(n=7)\end{array}$ & $\begin{array}{c}\text { Total } \\
(n=30)\end{array}$ \\
\hline 0 & & 3 & 6 & 4 & $13(43.4 \%)$ \\
\hline 1 & 1 & 1 & 3 & 1 & $6(20 \%)$ \\
\hline 2 & 1 & 1 & 2 & 6 & $10(33.3 \%)$ \\
\hline 3 & & & 1 & & $13(3.4 \%)$ \\
\hline
\end{tabular}

Some authors accept that the presence of breast tissue measuring at least $2 \mathrm{~cm}$ in diameter is sufficient for the diagnosis of gynecomastia [6,7], but others require the presence of breast tissue measuring at least $0.5 \mathrm{~cm}$ in diameter $[5,8]$. However, apart from the study by Ramadan et al [5], all these studies were conducted using clinical observations alone. Ramadan et al [5] found that a diameter of $5 \mathrm{~mm}$ was required for the diagnosis of gynecomastia in patients evaluated via US. In our study group, the minimum acceptable retroareolar tissue thickness was $3 \mathrm{~mm}$, and we suggest that US can reveal millimetric changes in the evaluation of gynecomastia. However, in 
our study group, there was no correlation between the Tanner stages established by US and retroareolar tissue thickness. In contrast, Ramadan et al found a strong positive correlation between these two variables. In our study, the fact that we found no correlation could be related to our small sample size.

Vessels are essential for the development of all tissues; therefore, evaluating them with color Doppler US in patients with gynecomastia might prove to be as important as using this diagnostic instrument to determine the Tanner staging system. We found a strong positive correlation between the Tanner stages and arterial and venous flow scores, as did Ramadan et al [5]. In our assessment of arterial flow, it was not detected in some cases, especially in the early Tanner stages (II, III), but a prominent arterial flow score was noted during the late Tanner stages (IV, V). The retroareolar region was the most vascular region of the breasts in our study group and in the study by Ramadan et al [5].

The RI is an important diagnostic tool for the evaluating vascular resistance in the different tissues. Knowing that vascularity plays an essential role in the development of all tissues, the RI could potentially serve as an independent, functional instrument for objectively evaluating gynecomastia. Our study was conducted with the aim of establishing an additional US tool for the evaluation of gynecomastia as it relates to the Tanner staging system, and we found a positive correlation between the Tanner stages and the mean RI values of the three breast regions in which vascularity was detected. In addition, Ramadan et al [5] also reported RI values on the same three breast regions, but they did not correlate the mean RI values of the breasts with the Tanner stages. In our study, the mean RI values of these three regions was 0.62 \pm 0.084 , and Ramadan et al [5] found similar values ( 0.61 $\pm 0.076)$. Furthermore, in the study by Rettenbacher et al [9] in which 200 healthy breasts were examined using color Doppler US, the mean RI values of premenopausal women was 0.64 , and the authors found that these values were slightly higher for postmenopausal women at 0.70 .

To our knowledge, this is the second study that has discussed the characteristics of gynecomastia using Doppler US, and it is believed to be the first study that has offered an alternative method to using US to determine the Tanner scoring system.

There were some limitations of this study. As previously mentioned, our sample size was small. In addition, no pathological investigation was conducted. However, it is not possible in clinical medicine to establish a diagnosis in all patients by means of histopathological examinations of biopsy specimens. In fact, it was not necessary to make a histopathological diagnosis for all of our patients when we considered that gynecomastia is a spontaneously reversible condition. Finally, all of our examinations were performed by a single radiologist, which decreased the value of our study.

\section{Conclusions}

Our results show that vascularity is correlated with breast development in patients with gynecomastia. Furthermore, it is possible that the RI could be as important as US in the establishment of Tanner stages. However, future studies with larger patient groups should be undertaken to define other potential diagnostic color Doppler US tools.

Conflict of interests: none.

\section{References}

1. Appelbaum AH, Evans GF, Levy KR, Amirkhan RH, Schumpert TD. Mammographic appearances of male breast disease. Radiographics 1999; 19: 559-568.

2. Garcia CJ, Espinoza A, Dinamarca V, et al. Breast US in children and adolescents. Radiographics 2000; 20: 16051612.

3. Einav-Bachar R, Philip M, Aurbach-Klipper Y, Lazar L. Prepubertal gynecomastia: aetiology, course and outcome. Clin Endocrinol (Oxf) 2004; 61: 55-60.

4. Braunstein G. Gynecomastia. In: Harris JR, Lipman ME, Morrow M, Helman S (eds). Diseases of the breast. Philadelphia, Lippincott-Raven, 1996: 54-60.

5. Ramadan SU, Gokharman D, Kaçar M, Koşar P, Koşar U. Assessment of vascularity with color Doppler ultrasound in gynecomastia. Diagn Interv Radiol 2010; 16: 38-44.

6. Lucas LM, Kumar KL, Smith DL. Gynecomastia. A worrisome problem for the patient. Postgrad Med 1987; 82: 7381.

7. Niewoehner CB, Nuttal FQ. Gynecomastia in a hospitalized male population. Am J Med 1984; 77: 633-638.

8. Nydick M, Bustos J, Dale JH Jr, Rawson RW. Gynecomastia in adolescent boys. JAMA 1961; 178: 449-454.

9. Rettenbacher T, Hollerweger A, Macheiner P, Gritzmann N. Color Doppler sonography of normal breasts: detectability of arterial blood vessels and typical flow patterns. Ultrasound Med Biol 1998; 24: 1307-1311. 